\title{
A study on microvascular density in breast carcinoma
}

\author{
Pyakurel D ${ }^{1}$, Karki S2, Agrawal CS \\ ${ }^{I}$ Department of Pathology and Laboratory Medicine, Grande International Hospital, Dhapasi, Kathmandu, Nepal \\ ${ }^{2}$ Department of Pathology, BP Koirala Institute of Health Sciences, Dharan, Nepal \\ ${ }^{3}$ Department of Surgery, BP Koirala Institute of Health Sciences, Dharan, Nepal
}

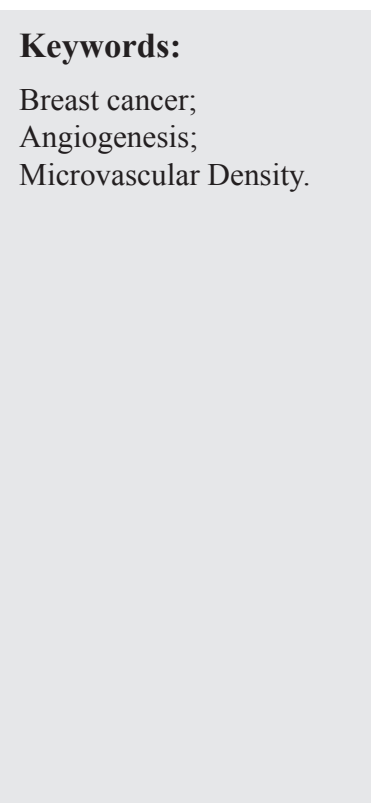

\begin{abstract}
Background: Breast Cancer is the most frequent neoplasm causing death in women between 35-55 years of age. Of the Prognostic indicators existing for breast cancer, axillary lymph node status has been regarded as the most important one. Twenty to thirty percent of all lymph node negative patients will still develop a recurrence of the disease within 10 years of initial treatment. Therefore, a new prognostic marker that could identify patients at high risk of tumor recurrence more accurately than existing indicators would be of great value, one potential indicator is tumor-induced angiogenesis.
\end{abstract}

Materials and Methods: This is a six months prospective (January 2010-June 2010) and 1 year retrospective (Jan2009-Dec2009) study which included thirty five breast cancer cases visiting the Surgical OPD. Angiogenesis was estimated by determining micro vessel counting after immune staining the paraffin embedded tissue sections using anti-CD34 antibody.

Results: Age of the patients ranged from 25 to 80 years with a mean age of 45.48 years. Most of the cases were infiltrating ductal carcinoma comprising of 33 cases $(94.28 \%)$. Three cases $(9.10 \%)$ showed vascular invasion by the tumor. Majority showed (63.64\%) vessel count of less than 200 per 10 high power fields.

Conclusion: Micro vascular density positively correlated with size of the tumor, lymph nodes involved by the tumor and Nottingham prognostic index. In the future, Antibodies specific to proliferating endothelium, together with the development of automated image analysis, may improve the accuracy and value of measuring angiogenesis-induced microvessel density.

\section{INTRODUCTION}

Breast Cancer is the most frequent neoplasm in women and the most frequent cause of death in women between 35-55 years of age. The introduction and development of new approaches to the management and treatment of breast cancer have highlighted the importance of patient's assessment, in order that optimal therapeutic treatment is

\author{
Correspondence: \\ Dr. Devish Pyakurel, MD \\ Department of Pathology and Laboratory Medicine, \\ Grande International Hospital, Dhapasi, Kathmandu, Nepal \\ E-mail:devishme@gmail.com
}

received. A number of established prognostic indicators exist for breast cancer, which include tumor size, lymph node status, histological grade, tumor type, vascular invasion and estrogen receptor status. Of these, axillary lymph node status has been regarded as the most important prognostic marker. However, $20-30 \%$ of all lymph-node-negative patients will still develop a recurrence of the disease within 10 years of initial treatment of the Primary tumor. Therefore, it is clear that a new prognostic marker that could identify patients at high risk of tumor recurrence more accurately than existing 
indicators would be of great value, and would allow more appropriate and effective treatment of those at greatest risk. One potential indicator in breast cancer is tumor-induced angiogenesis. ${ }^{1}$

Angiogenesis is the process which results in the growth and formation of new blood vessels. Angiogenesis is a prerequisite for tumor growth and metastasis. Neo vascularization provides not only the route for nutrient supply to the tumor but also the conduit for tumor cells to be shed into the circulation. New proliferating capillaries have leaky basement membranes, making them more accessible to tumor cells than mature vessels. It has been demonstrated that increasing density of newly formed micro vessels in growing tumors correlated closely with increasing number of tumor cells shed into the blood stream. In recent years, mounting evidence has suggested that quantification of intratumor microvessel density (MVD) by immunostaining for endothelial cell markers, such as CD34 $4^{1-13}$ CD31 $1^{1-3,13-16}$ von Willebrand factor (vWF) $)^{1-3,17,18}$ may be a useful prognostic predictor in cancer patients. A prognostic influence of MVD independent of conventional pathologic prognosticators has been demonstrated in a variety of cancers, such as breast carcinoma, gastric carcinoma, colorectal carcinoma, pancreatic carcinoma, testicular germ cell tumor, malignant melanoma, and even hematologic malignancies. However, results of studies on the prognostic value of MVD have not been homogeneous, probably because of factors such as methodological variation, selection bias in using different areas of tumors for study, and a lack of accurate patient follow-up data in retrospective studies. It has been emphasized that the prognostic significance of tumor MVD should be evaluated in a prospective manner with a standardized methodology. The introduction and development of new approaches to the management and treatment of breast cancer have highlighted the importance of patient's assessment, in order that optimal therapeutic treatment is received. Angiogenesis has attracted growing interest as a prognostic indicator in tumor progression. The theory is that the number of micro vessels within a tumor provides an estimate of the angiogenic potential of tumor cells and thus the probability of tumor growth, invasion, and metastasis. This relationship has been confirmed in multiple tumor types, including but not limited to, melanoma, prostate, and breast cancer. Tumor angiogenesis is generally measured by quantifying micro vessel density (MVD) in sections immune stained for vascular endothelial cell markers as CD34. ${ }^{1-13}$

In normal life, angiogenesis has a vital role in reproduction, embryogenesis, menstruation, and wound healing and repair. Its importance in solid tumors was first recognized by Folkman et al in 1971, when he suggested that the continued growth of tumors was dependent on angiogenesis. Micro vascular density is considered to be a marker of the neoangiogenetic process, which can be assessed on archival slides through the evaluation of immunoreactivity to some endothelial antigens $(\mathrm{CD} 34)^{1-13}$ a $115 \mathrm{~K}$ Da single chain trans membrane glycoprotein present on the surface of all endothelial cells. Over the last decade, assessment of angiogenesis has emerged as a potentially useful biological prognostic and predictive factor in human solid tumors. With the development of highly specific endothelial markers that can be assessed in histological archival specimens, several quantitative studies have been performed in various solid tumors.

The majority of published studies have shown a positive correlation between intra-tumoral micro vessel density, a measure of tumor angiogenesis, and prognosis in solid tumors.

This study aimed to find possible association of micro vascular density with other recognized prognostic factors such as size, lymph node status, type of tumor, grade and vascular invasion.

\section{MATERIAL AND METHODS}

A six months prospective (January 2010-June 2010) and 1 year retrospective (Jan2009-Dec2009) samples were included for the study. A minimum of thirty five samples during this period was included in the study. Angiogenesis was estimated by determining micro vessel counting after immune staining the paraffin embedded tissue sections using anti-CD34 mouse monoclonal antibody, employing avidin-biotin immune peroxidase technique. Immunohistochemical staining for CD 34 All archival paraffin blocks of mastectomy specimen of each case, histopathologically proven breast carcinoma were initially checked by hematoxylin and eosin stained slides to select a tissue block with tumor proper.

Microvessel counted manually under high power objectives of microscope following established procedure to obtain semi quantitative micro vascular density .The areas containing the greatest numbers of micro vessels or 'Tumor hotspots' was identified by scanning the stained sections at low magnification ( $x 40$ and $\mathrm{x} 100)$ using a light microscope, once these areas were recognized, individual stained micro vessel was counted at x200 magnification. Ten fields per tumor section was counted in the areas that appear to contain the greatest number of micro vessels on scanning at low magnification.

The results were expressed in terms of average micro vessel counts (MVC) per square millimeter of tissue area. Collected data were analyzed using SPSS version 10.

To assure quality control: Selection of appropriate slides (i.e. representing tumor proper) was chosen for staining with CD34, control slide with each selected case was run 
Table 1: Correlation of Micro vascular density and P values with other prognostic factors

\begin{tabular}{lccccc}
\hline PROGNOSTIC FACTORS & TUMOR SIZE & $\begin{array}{c}\text { AXILLARY LYPH NODE } \\
\text { STATUS }\end{array}$ & $\begin{array}{c}\text { TUMOR } \\
\text { GRADE }\end{array}$ & $\begin{array}{c}\text { VASCULAR } \\
\text { INVASION }\end{array}$ & NPI \\
PEARSONS CORRELATION & 0.11 & 0.316 & 6.027 & .099 & 0.496 \\
P VALUE & 0.065 & 0.074 & 0.006 & 0.755 & $0.003 *$ \\
$* \mathrm{p}<0.05$ & & & & &
\end{tabular}

and the micro vascular density count done once before final result were published.

Micro vascular density positively correlated with age of the patient, size of the tumor, lymph nodes involved by the tumor and total lymph nodes dissected out and Nottingham prognostic index but none of them show significant $P$ value except for NPI. Significant correlation is indicated at the 0.05 level, Microvascular density and Histologic grade showed near significant relation with a $P$ value of 0.006 . The $\mathrm{P}$ value was significant (.003) for micro vascular density and Nottingham prognostic index.

Microvascular density did not have a significant relationship (P value $>0.05$ ) with vascular invasion in this study although positively correlated (Table 1).

\section{RESULTS}

This was a hospital based study, of carcinoma breast cases. A total of thirty five mastectomy cases were included in this study. The study period was six months prospective and one year retrospective. Age of the patients enrolled in this study ranged from 25 years to 80 years with a mean age of 45.48 years. Number of patients older than 40 years comprised $23(69.69 \%)$ and less than 40 years comprised $10(30.31 \%)$. Most of the cases of breast cancer were seen between the ages of 35 to 55 years. Most of the cases in this study were of infiltrating ductal carcinoma comprising of 33 cases $(94.28 \%)$ followed by two cases of infiltrating lobular carcinoma $(5.72 \%)$. Histological grading was done in all the cases of infiltrating ductal carcinoma.14 (42.4\%) cases were found to have grade II disease followed by $11(33.3 \%)$ with grade III. Grade I disease was seen in 8 cases (24.2\%). Thirty cases in this study did not show vascular invasion by the tumor. Three cases $(9.10 \%)$ showed vascular involvement by the tumor. Representative sections from paraffin blocks were immunostained using anti CD34 mouse monoclonal antibody, employing Avidin Biotin immunoperoxidase technique. Areas of maximum number of CD34 expression (Angiogenesis) were identified by scanning with light microscopy at low power and the area of greatest micro vessel immunostaining was designated as "Hot Spots" (fig 1 \& 2).

Within the areas of maximum angiogenesis (Hot Spots) micro vessels were counted manually on a $200 x$ field $\left(1.05 \mathrm{~mm}^{2}\right)$. Any dark stained endothelial cell or cell clusters separate from adjacent structures were considered a single vessel. Large vessel with thick muscular wall and large vessel of lumen containing more than eight blood cells in diameter were excluded from the counts. The results of the count were expressed in terms of an average micro vessel count per square millimeter of tissue area $\left(\mathrm{vv} / \mathrm{mm}^{2}\right)$ and expressed as microvascular density. Micro vascular density was calculated at high power field in ten fields ranged from 3.8 to 29.3 (vv/mm2) with arithmetic mean value of $17.97(\mathrm{vv} / \mathrm{mm} 2)$ with a standard deviation of 7.147. Majority of the patients showed (63.64\%) vessel count of less than 200 per 10 high power fields. Micro vascular density positively correlated with size of the tumor, lymph nodes involved by the tumor and Nottingham prognostic index but none of them showed significant $\mathrm{P}$ value except for NPI. Microvascular density and Histologic grade showed near significant relation with a $P$ value of 0.006 . Micro vascular density do not have significant relationship ( $P$ value $>0.05$ ) with vascular invasion in this study although positively correlated.

\section{DISCUSSION}

Breast cancer is the leading cause of cancer deaths among women. Results from experimental studies suggest that tumor progression and metastasis in breast cancer is angiogenesis dependant. The College of American Pathologists has stated that further study of quantification of tumor angiogenesis is still required to demonstrate its prognostic value in breast cancer. Angiogenesis has attracted growing interest as a prognostic indicator in tumor progression. The theory is that the number of micro vessels within a tumor provides an estimate of the angiogenic potential of tumor cells and thus the probability of tumor growth, invasion, and metastasis. This relationship has been confirmed in multiple tumor types, including but not limited to, melanoma, prostate, and breast cancer. Tumor angiogenesis is generally measured by quantifying micro vessel density in sections immune stained for vascular endothelial cell markers, such as CD34, CD31 or Factor VIII. Takao Kato et al found the significant independent prognostic factor associated with long term survival in Japanese breast cancer patients, especially in node-negative patients. ${ }^{21}$

However, results of studies on the prognostic value of MVD have not been homogeneous, probably because of factors such as methodology variation, selection bias in using different areas of tumors for study, and a lack of 


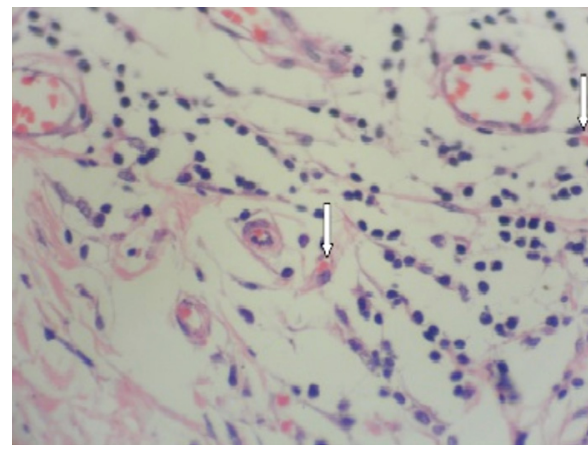

Figure 1: Blood vessels (arrows) seen in the tumor section (HE stain, X400).

accurate patient follow-up data in retrospective studies. It has been emphasized that the prognostic significance of tumor MVD should be evaluated in a prospective manner with a standardized methodology. Degree of vascularisation in different cancers and angiogenesis was heterogeneous within same tumor so micro vessels in areas of greatest micro vessel density "Hot spots"

were counted. We followed the method of counting vessels as described by Weidner et a ${ }^{16}$ by finding the vascular hotspots at low power and point count at 200x magnification. In contrast to the study done by L Martin et all we had taken one monoclonal antibody to CD34 and counted ten fields this would greatly reduce the chance of missing the most vascular area. Earlier studies only assessed one x 200 microscopic fields for micro vessel density and this was used for statistical analysis. We had taken the slide representing the sections from the tumor as L Martin et al concluded that the microvessel density measured in histological sections were representative of whole tumour vascularity. ${ }^{11}$

In a study done by H P Dhakal et $\mathrm{al}^{22}$ and Fox B et $\mathrm{al}^{23}$ the Chalkley method appears to be the better method in estimating the prognostic impact of vascularity in invasive breast carcinomas. Microscopic analysis for areas of the tumor that contained the most capillaries and small venules (micro vessels) good correlation between intra tumoral vascularization to accurately assess a particular tumor's angiogenic potential and found that visual scoring was superior hence we also followed this.

Since the original study by Weidner et al. in $1991^{16}$, other workers have looked at the prognostic significance of angiogenesis in breast cancer. In these studies, angiogenesis (measured by MVD) was compared to a number of clinico $\square$ pathological indicators, including tumour size, histological grade, estrogen receptor status, lymph node status, prognosis (disease $\square$ free survival and overall survival) and patient age.

In this study also we try to find any association of micro vascular density with prognostic factors as Tumor size, axillary lymph node status, Tumor type, histologic grade,

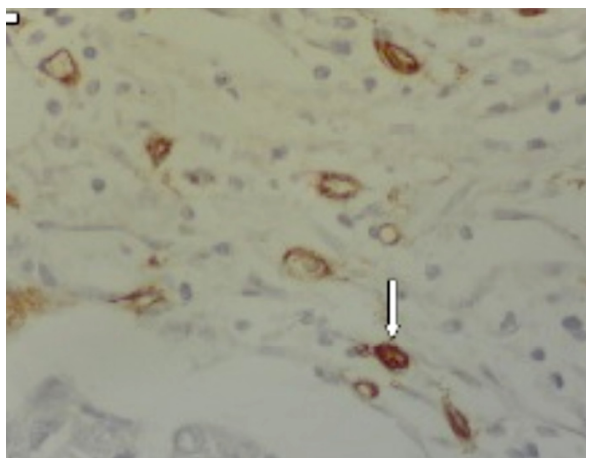

Figure 2: Microvessels highlighted with CD34 stain, X400 (arrowhead).

vascular invasion. In this study the mean and median of MVD was 17.97 with a standard deviation of 7.147 (average Range, 3.8 to 29.3) was found. The mean and median (range) microvessel counts from the Weidner et al16 study was 60 and 56 (range, 8 to 167), respectively, with a $0.74 \square$ mm2 counting area. In contrast, Van Hoef et al19 obtained higher ranges of 67 and 101 respectively, with a $0.476 \square$ $\mathrm{mm} 2$ counting area.

In contrast to the finding of Horak et al,14 this study could not find a significant relationship between micro vascular density and size of the tumor with $\mathrm{P}$ value of 0.65 . A borderline significant correlation $(\mathrm{P}=0.06)$ between increasedMicro vascular density and high $\square$ grade tumours was found by this study similar to that found by Horak et al,14 Weidner et al16 and Bosari et al.19

The MVD and lymph nodes involved were correlated in this study but the $\mathrm{P}$ value was 0.074 in contrast to the studies done by Horak et al14, and Weidner et al16 and Bosari et al19 who found a significant relationship between increased MVD and metastasis to lymph nodes.

Goulding et $\mathrm{al}^{13}$, did not find any relation between the two variables. As a prognostic indicator for breast carcinoma, Bosari et $\mathrm{al}^{19}$, Horak et $\mathrm{al}^{14}$ and Weidner et $\mathrm{al}^{16}$ found a significant difference in relapse free survival and/or overall survival rates between patients with high and low MVD. However, Goulding et $\mathrm{al}^{13}$, Van Hoef et $\mathrm{al}^{17}$, Costello et $\mathrm{al}^{18}$ failed to find any association between Micro vascular density and prognosis. In this study we found the MVD was increasing with age but this was not significant ( $\mathrm{P}$ value $=0.9$ ) this finding was similar to that found by Maxine Orre et al15. This study found positive correlation between MVD and prognosis but the $\mathrm{P}$ value was not significant (0.008) similar findings were present in the study done by Goulding et $\mathrm{al}^{13}$, Van Hoef et $\mathrm{al}^{17}$ and Costello et $\mathrm{al}^{18}$.

Clearly, it is apparent that there are many discrepancies between results obtained by different pathology laboratories, and a large number of these can be attributed to differences in the methods employed. The need for additional prognostic 
and predictive factors has led to identification of a plethora of potentially useful markers. ${ }^{24}$

When counting micro vessels, the selection of antibody for immunostaining is critical. The choice of antibody to detect endothelial cells is a compromise between the sensitivity and specificity of those available and a number of different antibodies have been used in the studies carried out, with no universal agreement on which is best. This is not altogether surprising as the sensitivity and specificity of all antibodies used are affected by the choice of fixative, tissue-processing regime, antigen-retrieval system and visualisation method. Angiogenesis research in breast carcinoma majority of the retrospective studies showed that angiogenesis was an important new prognostic indicator in early-stage breast carcinoma. This marker should be evaluated in prospective controlled clinical trials to demonstrate whether adjuvant therapies may improve the prognosis of those patients at high risk, e.g those with highly vascularized tumors. ${ }^{25}$

The vascular endothelial marker in the evaluation of microvascular density used in this study was CD34 which stained micro vessels greater and more intensely as was shown by a study done by and L martin et all in 1997 and Da silva BB et $\mathrm{al}^{2}$ in 2009.

The microvessel densities obtained by the Van Hoef17 group are in a range greater than what would be expected by using anti-CD31 to highlight microvessels, and Horak et $\mathrm{al}^{14}$ found anti-CD31 to be the most sensitive endothelial marker for highlighting intratumor microvessels. These discrepancies suggest methodological problems. The size and design of a study can also influence the results obtained. Most studies of tumour angiogenesis in breast carcinoma have used less than the 150 patients recommended for evaluating its usefulness as a prognostic and predictive indicator. In this study the number of patients was only thirty five. At present, micro vessel density as a prognostic marker for breast cancer is unreliable. Intratumoral vascularization appears to be an early event that is necessary but not sufficient for metastatic progression. Microvessel count seems to be an excellent marker to identify patients with good prognosis. ${ }^{27}$ Contradictions and inconsistencies have been demonstrated in the studies carried out, although the majority found that high MVD correlated with prognosis, histological grade and lymph node status. The reasons for these variations include the different investigation methods employed, technical problems (e.g. variability in immune staining) and the subjectivity involved in the process of selection and counting. In the future, however, antibodies specific to proliferating endothelium, together with the development of automated image analysis, may improve the accuracy and value of measuring angiogenesis-induced microvessel density.

\section{CONCLUSION}

Micro vascular density positively correlated with size of the tumor, lymph nodes involved by the tumor and Nottingham prognostic index. In the future, Antibodies specific to proliferating endothelium, together with the development of automated image analysis, may improve the accuracy and value of measuring angiogenesis-induced microvessel density.

\section{REFERENCES}

1. L Martin, B Green, C Renshaw, D Lowe, P Rudland, SJ Leinster, J Winstanley. Examining the technique of angiogenesis assessment in Invasive breast cancer. British journal of cancer 1997;76:1046-54. CrossRef

2. Da Silva BB, Lopes costa PV, dos Santos AR et al. Comparison of three vascular endothelial markers in the evaluation of micro vessel density in breast cancer. European journal of Gynecological Oncology 2009;30:285-8.

3. Siitonen SM, Haapasalo HK, Rantala IS, Helin HJ, Isola JJ. Comparison of different immunohistochemical methods in the assessment of angiogenesis: lack of prognostic value in a group of 77 selected node-negative breast carcinomas. Mod Pathology 1995;8:745-52. PMid:8539232

4. Hansen S, Grabau DA, Sørense FB et al. Vascular grading of angiogenesis: prognostic significance in breast cancer. British journal of cancer 2000;82:339-47. PMid:10646886

5. Murri AM, Hilmy M, Bell J et al. The relationship between the systemic inflammatory response, tumour proliferative activity, Tlymphocytic and macrophage infiltration, microvessel density and survival in patients with primary operable breast cancer. British journal of cancer 2008;99:1013-9. CrossRef

6. Popiela TJ, Sikora J, Klimek M et al. The analysis of CD 34 antigen immunoreactivity in invasive ductal breast cancer with respect to the presence of lymph node metastasis. European journal of gynecological oncology2009;30:285-8.

7. Heimann R, Ferguson D, Powers C, Recant WM, Weichselbaum RR, Hellman S. Angiogenesis as a predictor of long-term survival for patients with node-negative breast cancer. Journal of national cancer institute 1996;88:1764-9. CrossRef

8. Kanjanapanjapol S, Wongwaisayawan S, Phuwapraisirisan $\mathrm{S}$, Wilasrusmee C. Prognostic significance of microvessel density in breast cancer of Thai women. Journal of medical association Thai 2007;90:282-90. PMid:17375633

9. Li YJ, Yang L, Xia Q, Wen G. Hemodynamic changes in benign and malignant breast tumors and the mechanism. Nan FangYi Ke DaXue Xue Bao2009;29:1557-60. 
10. Kumar S, Ghellal A, Cheng Li, Ged Byrne, Najib Haboubi, Ji Min Wang, and Nigel Bundred Breast Carcinoma: Vascular Density Determined Using CD105 Antibody Correlates with Tumor Prognosis.Cancer research 1999;59:856-61. PMid:10029075

11. Ludovini V, Sidoni A, Pistola L, Bellezza G and De Angelis V. Evaluation of the Prognostic Role of Vascular Endothelial Growth Factor and Microvessel Density in Stages I and II breast cancer Patients. Breast Cancer Research and Treatment 2003;81:159-61. CrossRef

12. L Martin, C Holcombe, B Green, S J Leinster and J Winstanley. Is a histological section representative of whole tumour vascularity in breast cancer. British Journal of Cancer 1997;76:40-3. CrossRef

13. Goulding H, Abdul Rashid NF, Robertson JF, Bell JA, Elston $\mathrm{CW}$, Blamey RW, Ellis IO. Assessment of angiogenesis in breast carcinoma: an important factor in prognosis. Hum Pathol 1995;26:1196-200. CrossRef

14. Horak ER, Leek R, Klenk N, LeJeune S, Smith K, Stuart N, Greenall M, Stepniewska K, Harris AL. Angiogenesis, assessed by platelet/ endothelial cell adhesion molecule antibodies, as indicator of node metastases and survival in breast cancer. Lancet 1992;340:1120 4 CrossRef

15. Maxine Orre, Beatrice Susil and Peter A.W. Rogers Micro vesse density and vascular basement membrane immunostaining in tumours of the breast. Angiogenesis 1999;2:175-80. CrossRef

16. Weidner N, Semple JP, Welch WR, Folkman J. Tumor angiogenesis and metastasis -correlation in invasive breast carcinoma. N Engl Med 1991;324:1-8.

17. Van Hoef ME, Knox WF, Dhesi SS, Howell A, Schor AM. Assessment of tumour vascularity as a prognostic factor in lymph node negative invasive breast cancer. Eur J Cancer 1993;29:1141-5. CrossRef
18. Costello P, McCann A, Carney DN, Dervan PA. Prognostic significance of microvessel density in lymph node negative breast carcinoma. Hum Pathol 1995;26:1181-4. CrossRef

19. Bosari S, Lee AK, DeLellis RA, Wiley BD, Heatley GJ, Silverman ML. Microvessel quantitation and prognosis in invasive breast carcinoma. Hum Pathol 1992;23:755-61. CrossRef

20. Weidner N, Semple JP, Welch WR, Folkman J. Tumor angiogenesis a new significant and independent prognostic indicator in early stage breast carcinoma. N Engl J Med 1992;84:1875-87.

21. Takao Kato, Shingo Kameoka, Tsunehito Kimura, Naohiro Soga and Yutaka Abe. Angiogenesis as a predictor of long-term survival for 377 Japanese patients with breast cancer. Breast cancer Research and Treatment 2001;70:65-7. CrossRef

22. Dhakal HP, Bassarova A, Naume B et al. Breast carcinoma vascularity: a comparison of manual microvessel count and Chalkley count. Histopathol 2009;24:1049-59.

23. Fox SB, Leek RD, Weekes MP, Whitehouse RM, Gatter KC, Harris AL. Quantitation and prognostic value of breast cancer angiogenesis: comparison of microvessel density, Chalkley count, and computer image analysis. J Pathol 1995;177:275-83.CrossRef

24. Gasparini G, Pozza F, Harris AL. Evaluating the potential usefulness of new prognostic and predictive indicators in nodenegative breast cancer patients. J Natl Cancer Inst 1993;85:1206-19. CrossRef

25. Gasparini G, Harris AL. Clinical importance of the determination of tumor angiogenesis in breast carcinoma: much more than a new prognostic tool. J Clin Oncol 1995;13:765-82. PMid:7533829

26. Giampietro Gasparini. Biological and clinical role of angiogenesis in breast cancer. Breast cancer Research and Treatment 1995;36:3-10. CrossRef 